\title{
Genetic Analysis in the Diagnosis of Intellectual Disabilities in Adults
}

\author{
Lao Jose Ignacio ${ }^{2}$, Serrahima Joaquim ${ }^{1}$, Simó Pita ${ }^{1}$, Tañà Joan ${ }^{1}$, Àngels Ortega ${ }^{1^{*}}$ \\ ${ }^{* 1}$ Catalonia Foundation, Cerdanyola del Vallés, Barcelona, Spain \\ ${ }^{2}$ Genomic Genetic International, Av. Diagonal, Barcelona,Spain
}

"Corresponding author: Àngels Ortega, Catalonia Foundation, Cerdanyola del valles, Barcelona, Spain, Tel: +34 935805 227; Email: aortega@grupcatalonia.cat

Citation: Jose LI, Joaquim S, Pita S, Joan T, Angels O (2017) Genetic Analysis in the Diagnosis of Intellectual Disabilities in Adults. Int J Genom Data Min 01: 104. DOI: 10.29011/2577-0616.000104

Received Date: 6 March, 2017; Accepted Date: 18 March, 2017; Published Date: 25 March, 2017.

\begin{abstract}
Background: High-resolution technologies such as array Comparative Genomic Hybridization (aCGH) are changing the diagnostic approach to complex disorders as Intellectual Disability (ID) in such manner, that it is being advocated to be the first-tier genetic testing for the diagnostic protocol in ID patients. According to this premise, we conducted a study in a cohort of institutionalized adult ID patients.
\end{abstract}

Method: 121 institutionalized idiopathic ID adult patients were tested by analytical procedures as standard karyotype, metabolic analysis, DNA testing for Fragile $\mathrm{X}$ and aCGH array. aCGH array was carried out from genomic DNA purified from blood samples by applying the Neuroarray technology (Genetadi, Bilbao, Spain).

Results: We identified a genetic cause in $80 \%$ of our sample where $71 \%$ of them have cryptic chromosomal abnormalities, 4\% Fragile X syndrome (FRAXA) and 5\% Inborn Errors of Metabolism (IEM). There is a predominance of microdeletions over microduplications being the $17 \mathrm{q}$ microdeletion the most frequent cryptic chromosomal abnormality detected in our sample.

Conclusion: According to this study we suggest that applying the aCGH technology as a first step within a diagnostic algorithm we will able to increase the diagnostic accuracy reducing the rate of idiopathic forms.

Keywords: Cryptic Chromosomal Abnormality; Fragile X; Inborn Errors of Metabolism; Intellectual Disability; Mental Retardation; Microdeletion; Microduplication; aCGH Array; 17q Microdeletion

\section{Introduction}

Intellectual Disability (ID, previously known as known as mental retardation) is a condition included in the neurodevelopment disorder group which is characterized by significant limitations in both intellectual functioning and in adaptive behavior covering many everyday social and practical skills having an onset prior to 18 years of age [1]. The reported prevalence of ID ranges between 1 and 3\% being higher among male versus female [2]. It depends on many variables as population (age, gender, social class and ethnicity) and criteria and methods of assessment used [2,3]. The cause of most ID patients is unknown. In fact, there are a het- erogeneous group of factors associated with increased risk of ID. Basically, these factors are classified as genetic and non-genetic factors with different contribution depending on each particular context and the moment of impact [4]. The most frequent genetic conditions are represented by chromosomal abnormalities followed by single-gene defects where inborn errors of metabolism are also included [5-7]. Among the non-genetic factors, we have to consider the moment of their impact: being of prenatal, perinatal or postnatal influence. Prenatal non-genetics factors are mainly represented by viral infections (e.g. toxoplasmosis, rubella) [8], drugs and toxic influences (e.g. alcohol) [9], dietary factors as iodine deficiency [10] and Rh incompatibility [11]. Among perinatal non-genetics factors the most outstanding are prematurity and a traumatic labor delivery which induces anoxia and brain damage [12], whereas among the most relevant postnatal influences are infections [13,14], poisonings, cerebral trauma (head injury) [15] or 
sociocultural factors [16].

In spite of that fact, in any case and independently of the stage we will always be in front of the dilemma to discern the real contribution of genetic and non-genetic variables in each patient in order to ensure the best genetic counseling for the family [17]. In general, it is estimated a recurrence risk of 3-9\% for families with one previous child with severe intellectual disability [18]. However, this risk has to be adjusted according to each specific condition. At this point, genetic analysis is an indispensable tool to clarify the causes related to family risks.

\section{Genetic Approach}

When considering genetic factors as cause of ID, it is important to recognize that approximately $10-40 \%$ of patients have a detectable chromosome abnormality [19]. Most of them show a recognizable pattern of clinical characteristics (distinguishing phenotype) which makes possible to suppose the diagnosis (e.g. Down syndrome or trisomy 21). However, many cases may not be so obvious to be clinically recognizable and we have to resort to algorithms designed to facilitate the diagnostic process. Taking into account those considerations, after an exhaustive clinical evaluation, the first test to be indicated is the chromosomal analysis (Karyotyping). Other patients, some of them with an atypical phenotype, may have small chromosomal rearrangements (deletions or duplications) non-detectable by karyotype which is contributing to an upward revision of chromosomal involvement in ID etiology [20].

Nevertheless, conventional chromosome analysis or karyotype has a limited resolution since it is only able to detect defects larger than $3 \mathrm{Mb}$ [21]. There are some other techniques to look for smaller chromosomal abnormalities as Multiplex LigationDependent Probe Amplification (MLPA) which is used to test sub telomeric regions of all chromosomes or like Fluorescent in Situ Hybridization (FISH) in order to confirm a clinical diagnosis of some well-known microdeletion/microduplication syndromes (e.g. Williams-Beuren syndrome, DiGeorge syndrome). Both time-consuming techniques are also carried out in specialized laboratories which combine conventional and molecular cytogenetics [22,23].

The chromosome microarray or comparative genomic hybridization technique (array-CGH) is the most recent technique to be used in genetic study of ID. It has the advantage to incorporate in a unique test the ability to identify microdeletions and/or microduplications with the highest degree of sensitivity (25-50 kb) [21]. In this sense, array-CGH is pointing out that it is necessary an upward recalculation of the frequency of chromosomal abnormalities in ID $[24,25]$. By definition a Copy Number Variations (CNVs) is an increased or decreased number of copies of a DNA fragment causing a deviation from the normal diploid pattern. There are CNVs which represent benign variations whereas others are responsible of a clinical phenotype [26]. Most of detected CNVs are de novo but some of them may be inherited from a parent. This is an important point to clarify to assure a correct genetic counseling to the family [27].

In spite of chromosomal causes, there are also monogenic causes explaining some of the inherited forms of ID [28]. The most common inherited form of monogenic ID, particularly in males, is the Fragile $X$ syndrome [29,30]. Its prevalence is 1 in 3,600 in males and approximately 1 in 4,000 to 6,000 in females. The defective gene is the FMR1 which is located in the long arm of chromosome X (Xq27.3) [31]. Most cases, especially adults, are easily identifiable by clinical examination because their peculiar phenotype (Martin-Bell phenotype), nevertheless, it is important to confirm the diagnosis by mean of the DNA testing to evaluate the FMR1 status following the guidelines defined by the American College of Medical Genetics (ACMG) [32].

There are many other X-linked and autosomal monogenic causes of ID. In chromosome $\mathrm{X}$ are described more than 80 gene defects associated with ID [33] and in monogenic chromosomes there are described more than 1400 genes and the list is still increasing for both syndromic and non-syndromic forms due to the latest technological improvements [28]. Metabolic disorders, which are also monogenic in origin, are another important cause of intellectual disabilities to be considered in diagnosis protocol applied to idiopathic ID [34]. Maybe its relevance was more pronounced in the past because today neonatal screenings are reducing their impact on this condition [35].

\section{Subjects and Methods}

\section{Subjects}

In the present study were included 121 adults (77 men, 44 women; age range 20-70 yrs.) with intellectual disabilities and dysmorphic pattern (in all of them are present abnormalities in cranium and face as well as hands and feet). They are all residing in an institution for the mentally retarded people ("Group Catalonia", Barcelona, Spain) with an average time of institutionalization of 19,7 years.

\section{Data Collection}

Clinical records and institutional archives were searched to collect data from each patient. Data were selected from diagnosis collected during 2013 with the objective to know possible etiologies of those patients who were previously classified as unknown etiologies. All patients were examined by one physician with expertise in clinical genetics and by clinical psychologists to delimit the clinical phenotype to be included in the study.

\section{Inclusion Criteria}

Three groups of patients were included according to the following characteristics: 
Citation: Jose LI, Joaquim S, Pita S, Joan T, Angels O (2017) Genetic Analysis in the Diagnosis of Intellectual Disabilities in Adults. Int J Genom Data Min 01: 104.

- Patients with moderate-severe intellectual disabilities and dysmorphic features of unknown etiology.

- $\quad$ Patients with moderate-severe intellectual disabilities and genetic diagnosis.

- Undiagnosed patients with clinical features suggesting a recognizable intellectual disabilities syndrome.

\section{Laboratory Investigations}

\section{Chromosome Analysis}

Chromosome preparations from peripheral blood cultures and cytogenetic analyses were performed using a high-resolution karyotype (850 bands).

\section{FMR1 Molecular Analysis}

The presence of an FMR1 gene expansion was analysed using molecular PCR procedures followed by fragment analysis by capillary electrophoresis (AmplideXtm FMR1PCRKit).

\section{Metabolic Studies}

An analytical study on dried blood of metabolic markers (analytes) was performed using an expanded neonatal screening test based on the tandem mass spectrometry (MS/MS) technology (NEONATAL-ONE®, Genetadi) which covers 56 metabolic disorders.

\section{CNV Analysis}

A blood sample ( 5 milliliters) in a tube with EDTA from each patient was analyzed by the aCGH microarrays technology Neuroarray (Genetadi, Bilbao, Spain). This technology is able to detect more than 900 regions associated to mental idiopathic and other neurodevelopment illnesses. To calculate the detection rate of the present study we determined the percentage of genetic defect carriers whilst the diagnostic yield is only referred to the percentage of patients carrying a clinically relevant $\mathrm{CNV}$.

Clinical relevance of detected CNV was assessed by accessing to database through the Ensemble Resources as DECIPHER (Database of Chromosomal Imbalance and Phenotype in Humans), the ISCA database [http://dbsearch.clinicalgenome.org/search/], and the OMIM [Mendelian Inheritance in Man].

\section{Results}

In the present study, we identified (Table 1) a genetic defect in 97 patients ( $80 \%$ of our sample), where 86 cases $(71 \%)$ have a cryptic chromosomal abnormality: $72 \mathrm{CNVs}$ on autosomal chromosomes and 14 on $\mathrm{X}$ chromosome, 5 patients (4\%) were Fragile X syndromes (FRAXA) and 6 cases (5\%) showed a metabolic disturbance. It was not possible to detect a genetic defect in 24 patients (20\%) (Figure 1).

\begin{tabular}{|c|c|c|c|c|}
\hline \multicolumn{2}{|c|}{$\mathbf{N}$} & $\%$ & \multicolumn{2}{|c|}{ DIAGNOSIS } \\
\hline \multirow{2}{*}{86} & 72 & \multirow{2}{*}{$71 \%$} & $\begin{array}{l}\text { autosomal } \\
\text { chrom. }\end{array}$ & $\begin{array}{l}\text { cryptic } \\
\text { chromo- }\end{array}$ \\
\hline & 14 & & X chrom. & $\begin{array}{c}\text { somal } \\
\text { abnormality }\end{array}$ \\
\hline \multicolumn{2}{|c|}{5} & $4 \%$ & \multicolumn{2}{|c|}{$\begin{array}{l}\text { fragile X syndromes } \\
\text { (FRAXA) }\end{array}$} \\
\hline \multicolumn{2}{|c|}{6} & $5 \%$ & \multicolumn{2}{|c|}{ metabolic disturbance } \\
\hline \multicolumn{2}{|c|}{24} & $20 \%$ & \multicolumn{2}{|c|}{ no genetic defect } \\
\hline \multicolumn{2}{|c|}{121} & & \multicolumn{2}{|c|}{ TOTAL } \\
\hline
\end{tabular}

Table 1

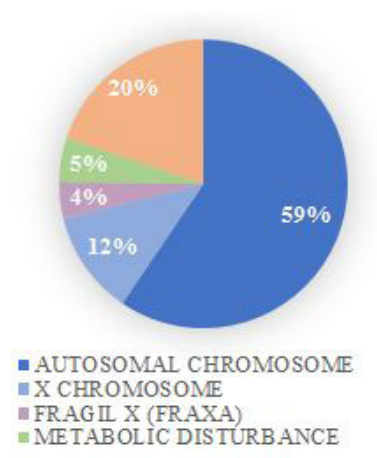

Figure 1: General Results.

The detection rate of our diagnosis protocol was $80 \%$ to reach to a diagnostic yield of $71 \%$ using the aCGH. There is a predominance of alterations in autosomal chromosomes $(83 \%)$ with respect to the sex chromosomes (17\%). Regarding to sex chromosomes, alterations are more frequent in the $\mathrm{X}$ chromosome than in chromosome $\mathrm{Y}$ in a relation of 12:2 respectively. By delving into the autosomal causes, we observed that chromosomes with a greater involvement are chromosome 17 (14 cases), chromosomes 2 and 9 (9 cases) and chromosomes 3 and 6 (Figure 2).

FIGURE 2. CRYPTIC CHROMOSOMAL ABNORMALTYY

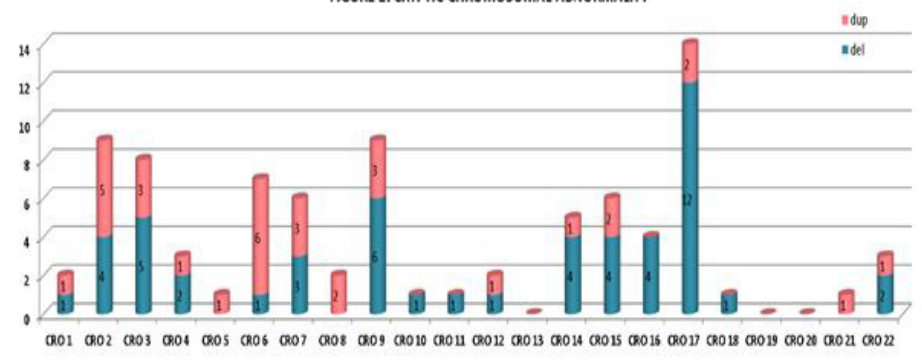

Figure 2: Cryptic Chromosomal Abnormality.

On the other hand, there is a predominance of microdeletions over microduplications (52 vs. 34 respectively) being the $17 \mathrm{q}$ microdeletion the most frequent cryptic chromosomal abnormality detected in our sample (10 cases; $13 \%$ of total detected CNVs). There are additional cryptic copy number alterations without clini- 
cal relevance (because they were previously reported in a normal population being catalogued as "benign CNVs") which were identified in 11 cases of our sample (9\%). Curiously, there is also a predominance of benign CNVs in the chromosome 17 (8 subjects).

\section{Fragile X Analysis}

The numbers of CGG repeats less than 50 were detected in 116 cases. CGG repeats in a full mutation range (more than 200 CGG) were detected in 5 male patients. All these patients showed a clinical phenotype coincident with the Martin-Bell phenotype.

\section{Metabolic Findings}

Metabolic defects were detected in 6 of 121 patients (5\%):

- $\quad$ Maple Syrup Urine Disease (MSUD)

- $\quad$ Phenyl Keton Uria (PKU)

- $\quad$ Urea Cycle Disorders (UCDs).

\section{Discussion}

Chromosomal abnormalities are the most common cause of intellectual disabilities in our patients. Coincident with our results is important to note that other recent scientific studies have detected that $40 \%$ of the chromosomal rearrangements apparently balanced using karyotype or FISH, end-up resulting in an unbalanced micro rearrangement after a high-resolution analysis using aCGH microarrays [21,25,26].

Microdeletions were the most frequent type of chromosomal defects detected in our sample suggesting that microdeletions might be more pathogenic than microduplications. This is also consequent with the published results by other authors on this regard [24,27]. In this sense, it is curious to note that the most frequent non-pathogenic cryptic rearrangements we found in our sample are represented by microduplications.

Among the 86 cases with probably pathogenic CNVs, there is a predominance of CNVs which have been previously associated with intellectual disabilities syndromes (MR-associated CNVs) being coincident with some Decipher known syndromes [36]. On the other hand, there are 11 cases with CNVs which are currently of uncertain clinical significance. Due to the characteristics, inherent to our sample (institutionalized adult patients) it was not possible to analyze their inheritance in a family study.

Nevertheless, neither of them has been previously described in normal population nor associated with Decipher known syndromes (https://decipher.sanger.ac.uk/syndromes\#syndromes/ overview). To understand the clinical relevance of a CNV we have to consider many standardized criteria together with the particular clinical evaluation. In addition of being a CNV that have not been observed among healthy individuals, in the first place, we have to consider the CNV size. The larger the CNV the more likely to encompass functional DNA sequences with an impact over the clinical phenotype [37]. In our study, we consider to analyze in depth as MR-associated CNV those CNVs with more than $100 \mathrm{~kb}$ which represent the $88 \%$ in our sample.

However, it is important to take into account that the relevance of the gene content might be not only related to the size which mean that quantity of genes is not the unique factor to consider, because quality also matter. There are shorter CNVs containing few genes but having a critical relevance regarding to the analyzed phenotype and in this sense, they might be more relevant than larger CNVs which have several non-relevant DNA sequences. That means that both quantity and quality should always be carefully assessed in order to decide the real significance of each analyzed CNV [38].

Another relevant criterion to consider is the inheritance using family information. In our study, we have 2 families with affected relatives sharing the same CNV which is smaller than $50 \mathrm{~kb}$ in both cases. There are 2 affected brothers with the same CNV of $13,3 \mathrm{~kb}$ in chromosome 16 (microdeletion at 16q24.1) in one of the families and two affected relatives (brother and sister) in the other family showing an $11,8 \mathrm{~kb} \mathrm{CNV}$ at chromosome 14 (microdeletion at $14 \mathrm{q} 22.1)[39,40]$.

Regarding to the gene content, it is curious to mention that the CNV at chromosome 14 affects the gene PTGER2. This gene codes for the EP2 receptor subtype of prostaglandin E2 (PGE2) functioning as a regulator of several inflammatory and neuroprotective processes. In animal models (knockout mice), it was determined that PTGER2 acts as a regulator of synaptic transmission in the hippocampus [41]. The other CNV at 16q24.1 affects the gene ATP2C2 which is a known candidate gene to have an important neurobiological significance [42].

Our study gives additional support to affirm that chromosomal microarray has to be included as the first clinical diagnostic test for patients with intellectual disabilities. This statement is in concordance with a consensus recently recommended by other researchers worldwide $[43,44]$. We propose to use a different analytical algorithm for Intellectual Mental Retardation (IMR) where the comparative genomic hybridization microarrays (aCGH) might be used as the first option after clinical valuations.

\section{Conclusion}

The present study confirms that genetics is an important factor to take into account in front of severe/profound mental retarded patient with dysmorphic pattern. According with that, CNVs are highly represented in patients with intellectual disabilities and dysmorphic pattern, being the most relevant genetic cause present in our population. The most common CNVs detected in our study related to intellectual disabilities are the microdeletions. The $17 \mathrm{q}$ microdeletion syndrome is distinguished as the most prevalent syndrome present in our patients. According to our findings, we consider that it is highly recommended to use the aCGH microarray technology as the first analytical test to carry out as part of the diagnostic protocol of MR patients. 
Citation: Jose LI, Joaquim S, Pita S, Joan T, Angels O (2017) Genetic Analysis in the Diagnosis of Intellectual Disabilities in Adults. Int J Genom Data Min 01 : 104.

\section{Limitations of present study}

Due to the particularities of the subjects included in the present study: institutionalized adult patients with a mean age of 45 years and a mean time of institutionalization of 19.7 years, there was not possible to carry out the corresponding familial testing analysis for more of them.

\section{References}

1. American Psychiatric Association (2013) Diagnostic and statistical manual of mental disorders. 5th ed. DSM-5. Washington DC.

2. Leonard H, Wen X (2002) The epidemiology of mental retardation: challenges and opportunities in the new millennium. HYPERLINK "https://www.ncbi.nlm.nih.gov/pubmed/12216056"Men Retard Dev Disabil Res Rev 8: 117-134.

3. Yeargin-Alsopp M, Boyle C, Braun K, Trevathan E (2008) The epidemiology of developmental disabilities. In: Accardo PJ (Ed) Neurodevelopmental Disabilities in Infancy and Childhood. 3rd edn. Paul H. Brooks Publishing Co, Baltimore: pp61-pp104.

4. Hayiou-Thomas ME (2008) Genetic and environmental influences on early speech, language and literacy development. J commun Disord 41: 397-498.

5. Rauch A, Hoyer J, Guth S, Zweier C, Kraus C, et al. (2006) Diagnostic yield of various genetic approaches in patients with unexplained developmental delay or mental retardation. Am J Med Genet A 140: 2063-2074.

6. Ropers HH (2008) Genetics of intellectual disability. Curr Opin Genet Dev 18: 241-250.

7. Raymond FL (2010) Monogenic Causes of Mental Retardation. Knight SJL (ed) Genetics of Mental Retardation. Monogr Hum Genet Basel, Karger 18: 89-100.

8. Torgerson PR, Mastroiacovo P (2013) The global burden of congenital toxoplasmosis: A systematic review. Bull World Health Organ 91: 501-508.

9. Morén C, Hernández S, Guitart-Mampel M, Garrabou G (2014) Mitochondrial Toxicity in Human Pregnancy: An Update on Clinical and Experimental Approaches in the Last 10 Years. Int $\mathrm{J}$ of Environ Res Public Health 11: 9897-9918.

10. Skeaff SA (2011) lodine Deficiency in Pregnancy: The Effect on Neurodevelopment in the Child. Nutrients 3: 265-273.

11. Geier DA, Mumper E, Gladfelter B, Coleman L, Geier MR (2008) Neurodevelopmental Disorders, Maternal Rh-Negativity, and Rho(D) Immune Globulins: A Multi-Center Assessment. Neuro Endocrinol Lett 29: $272-280$.

12. Maroney DA (2003) Recognizing the Potential Effect of Stress and Trauma on Premature Infants in the NICU: How are Outcomes Affected? Journal of Perinatology 23: 679-683.

13. Chau V, Brant R, Poskitt KJ, Tam EWY, Synnes A, et al. (2012). Postnatal infection is associated with widespread abnormalities of brain development in premature newborns. Pediatr Res 71: 274-279.

14. Bilder DA, Pinborough-Zimmerman J, Bakian AV, Miller JS, Dorius JT, et al. (2013) Prenatal and perinatal factors associated with intellectual disability. AM J Intellec Dev Disabil 118: 156-176.
15. Sophie Su YR, Veeravagu A, Grant G (2016) Neuroplasticity after Traumatic Brain Injury. In: Laskowitz D, Grant G, editors. Translational Research in Traumatic Brain Injury. Boca Raton (FL): CRC Press/Taylor and Francis Group.

16. Leighton $\mathrm{AH}$, Hughes JM (2005) Cultures as a Causative of Mental Disorder. The Milbank Quarterly 83: 10-11.

17. Lao Jl (2001) Diagnosis and genetic counseling in mental retardation. Rev Neurol 33:15-65.

18. Karam SM, Riegel M, Segal SL, Félix TM, Barros AJD, et al. (2015). Genetic causes of intellectual disability in a birth cohort: A populationbased study. Am J Med Genet A 167: 1204-1214.

19. Tzschach A, Ropers HH (2007) Genetics of Mental Retardation. Dtach Artebl 104: 1400-1405.

20. Menten B, Maas N, Thienpont B, Buysse K, Vandesompele J, et al. (2006) Emerging patterns of cryptic chromosomal imbalance in patients with idiopathic mental retardation and multiple congenital anomalies: a new series of 140 patients and review of published reports. J Med Genet 43: 625-633.

21. Di Gregorio E, Savin E, Biamino E (2014) Large cryptic genomic rearrangements with apparently normal karyotypes detected by arrayCGH. Mol Cytogenet 7: 82-92.

22. Schouten JP, McElgunn CJ, Waaijer R (2002) Relative quantification of 40 nucleic acid sequences by multiplex ligation-dependent probe amplification. Nucleic Acids Res 30: e57.

23. Flint J, Knight S (2003) The use of telomere probes to investigate submicroscopic rearrangements associated with mental retardation. Curr Opin Genet Dev 13: 310-316.

24. Devriendt K, Vermeesch JR (2004) Chromosomal phenotypes and submicroscopic abnormalities Human Genomics 1: 126-133.

25. Hochstenbach R, van Binsbergen E, Engelen J (2009) Array analysis and karyotyping: work flow consequences based on a retrospective estudy of 36,325 patients with idiopathic developmental delay in the Netherlands. Eur J Med Genet 52: 161-169.

26. Schluth-Bolard C, Delobel B, Sanlaville D, Boute O, Cuisett JM, et.al. (2009) Cryptic genomic imbalances in de novo and inherited apparently balanced chromosomal rearrangements: array CGH study of 47 unrelated cases. Eur J Med Genet. 52: 291-296.

27. Sykulski M, Gambin T, Bartnik M, Derwińska K, Wiśniowiecka-Kowalnik $\mathrm{B}$, et al. (2013) Multiple samples aCGH analysis for rare CNVs detection. J Clin Bioinforma 3: 12

28. Raymond FL (2010) Monogenic Causes of Mental Retardation: Genetics of Mental Retardation. Monogr Hum Genet:Basel, Karger 18: 89-100.

29. Artigas-Pallares A, Brun C, Gabau E (2001) Aspectos médicos y neuropsicológicos del Síndrome de X Frágil. HYPERLINK "http://ardilladigital.com/DOCUMENTOS/DISCAPACIDADES/X\%20FRAGIL/ Aspectos $\% 20$ medicos $\% 20 \mathrm{y} \% 20$ neuropsicologicos $\% 20 \mathrm{del} \% 20 \mathrm{sin}$ drome $\% 20$ X $\% 20$ fragil\%20-\%20Artigas $\% 20 y \% 20$ otros $\% 20-\% 20$ articulo.pdf'Rev Neurol Clin 2: 42-54.

30. Hunter J, Rivero-Arias O, Angelov A, Kim E, Leal J, et al. (2014) HYPERLINK "https://www.ncbi.nlm.nih.gov/pubmed/24700618"Epidemiology of fragile $X$ syndrome: A systematic review and meta-analysis. Am J Med Genet 164A: 1648-1658. 
Citation: Jose LI, Joaquim S, Pita S, Joan T, Angels O (2017) Genetic Analysis in the Diagnosis of Intellectual Disabilities in Adults. Int J Genom Data Min 01: 104.

31. Bardoni B, Mandel JL, Fisch GS (2000) FMR1 gene and fragile X syndrome. Am J Med Genet 97: 153-163.

32. Monaghan KG, Lyon E, Spector EB (2013) ACMG Standards and Guidelines for fragile $X$ testing: a revision to the disease-specific supplements to the Standards and Guidelines for Clinical Genetics Laboratories of the American College of Medical Genetics and Genomics. Genet Med 15: 575-586.

33. Ropers HH, Hamel BCJ (2005) X-linked mental retardation. Nat Rev Genet 6: 46-57.

34. Kahler SG, Fahey MC (2003) Metabolic disorders and mental retardation. Am J Med Genet C Semin Med Genet 117C: 31-41.

35. Van Karnebeek CDM, Stockler S (2012) Treatable inborn errors of metabolism causing intellectual disability: A systematic literature review. Mol Genet Metab 105: 368-381.

36. Webber C, Hehir-Kwa JY, Nguyen DQ, de Vries BB, Veltman JA, et al. (2009) Forging links between human mental retardation-associated CNVs and mouse gene knockout models. PLoS Genet 5: e1000531.

37. Kearney HM, Thorland EC, Brown KK, Quintero-Rivera F, South ST (2011) Working Group of the American College of Medical Genetics Laboratory Quality Assurance Committee American College of Medical Genetics standards and guidelines for interpretation and reporting of postnatal constitutional copy number variants. Genet Med 13: 680-685.

38. Rosenfeld J A, Coe B P, Eichler E E, Cuckle H, Shaffer L G (2013) Estimates of penetrance for recurrent pathogenic copy-number variations. Genet Med 15: 478-481.
39. Deak K L, Horn S R, Rehder C W (2011) The evolving picture of microdeletion/microduplication syndromes in the age of microarray analysis: variable expressivity and genomic complexity. Clin Lab Med 31: 543-564.

40. Lumaka A, Van Hole C, Casteels I, Ortibus E, De Wolf V, et al. (2012) Variability in expression of a familial $2.79 \mathrm{Mb}$ microdeletion in chromosome 14q22.1-22.2. Am J Med Genet A 158A: 1381-1387.

41. Jiang J, Dingledine R (2013) Prostaglandin receptor EP2 in the crosshairs of anti-inflammation, anti-cancer, and neuroprotection. Trends in Pharmacological Sciences 34: 413-423.

42. Smith AW, Holden KR, Dwivedi A, Dupont BR, Lyons MJ (2015) Deletion of 16q24.1 supports a role for the ATP2C2 gene in specific language impairment. J Child Neurol 30: 517-521.

43. Miller DT, Adam MP, Aradhya S, Biesecker LG, Brothman AR, et al. (2010) Consensus statement: chromosomal microarray is a first-tier clinical diagnostic test for individuals with developmental disabilities or congenital anomalies. Am J Hum Genet 86: 749-764.

44. Battaglia A, Doccini V, Bernardini L, Novelli A, Loddo S, et al. (2013) HYPERLINK "https://www.ncbi.nlm.nih.gov/ pubmed/23711909"Confirmation of chromosomal microarray as a firsttier clinical diagnostic test for individuals with developmental delay, intellectual disability, autism spectrum disorders and dysmorphic features. Eur J Paediatr Neurol 17: 589-599. 